\title{
Racionalismo y regeneración nacional en La Ciudad de los Reyes
}

\author{
Carlos García-Bedoya M. \\ Departamento Académico de Literatura
}

\section{Introducción}

La literatura peruana, lamentablemente, sigue siendo en gran medida un territorio inexplorado. Esto es particularmente cierto con respecto al siglo XIX e incluso comienzos del XX. Problamente pues no es un caso único el del injusto olvido en que yace sepultada una novela como La Ciudad de los Reyes, de Pedro Dávalos y Lissón (1863-1942). Más conocido por su labor como historiador ${ }^{1}$ Dávalos es sin embargo autor de seis novelas: La Ciudad de los Reyes (La Habana: Imprenta Avisador Comercial, 1906)²; Leguia (Lima: Librería e Imprenta Gil, 1915, 2 tomos) ${ }^{3}$, continuación muy inferior de la anterior; Manuel Pardo (Lima: Librería e Imprenta Gil, t. I, 1915; t. II, 1916); San Martín (Barcelona: Montaner y Simón, 1924)4, Bolívar (Barcelona: Montaner y Simón, 1924), continua ción de la anterior: Mercedes (Lima: Librería e Imprenta Gil, 1939). Algunas, como San Martín y Bolivar, son novelas históricas, muy en la huella de los Episodios Nacionales de Galdós (ambas llevan el

1 Su trabajo histórico apreciado es La primera centuria. Causas geográficas, políticas y económicas que han detenido el progreso moral y material del Perú en su primer siglo de vida independiente, 4 tomos, publicados entre 1919 y 1926.

2 Reeditada en 1989 en Lima por el Instituto Nacional de Cultura. Lamentablemente esa edición no tuvo una adecuada difusión y además no va acompañada de ningún prólogo o estudio que destaque sus méritos. Según se señala en esa reedición, existiría una primera edición de 1904 de la cual no tenemos otra información.Tanto Raúl Porras en sus Fuentes históricas peruanas (Lima: Juan Mejia Baca y P.L. Villanueva, 1954), como Elsa Villanueva de Puccinelli en su Bibliografia de la novela peruana (Lima: Ediciones de la Biblioteca Universitaria, 1969), y Jorge Basadre en su Introducción a las bases documentales para la historia de la República del Perú con algunas reflexiones (Lima: P.L. Villanueva, 1971) mencionan solamente la edición de 1906.

3 En esas misma edición se señala que se trata de la segunda edición. No tenemos información sobre la posible primera edición. Porras menciona una edición de 1913, aunque no da más datos. Tanto Elsa Villanueva de Puccinelli como Basadre sólo mencionan la edición de 1915.

4 Según Elsa Villanueva, habría una edición de Lima, publicada por Librería e Imprenta Gil en 1922. Ni Porras, ni Basadre mencionan tal edición.

LETRAS (Lima), 94: 135-146,1997. 
subtítulo explícito de Episodio de la independencia peruana). Otras, como Manuel Pardo y La Ciudad de los Reyes, sin dejar de lado el aspecto histórico -un pasado más reciente- incoporan también aportes de las denominadas Novelas Contemporáneas, del mismo Galdós.

La Ciudad de los Reyes merece ocupar un puesto destacado en la historia de la novela peruana, en particular en el titubeante periodo inicial que se prolonga hasta ya bien entrada la década del veinte del presente siglo. Lamentablemente, la crítica no le ha concedido mayor atención. Luis Alberto Sánchez le dedica unas pocas líneas en su La literatura peruana ${ }^{5}$ insistiendo en las deficiencia de las novelas de Dávalos, pero sin dejar de reconocerles cierto interés. Tamayo ni siquiera menciona a Dávalos en su Literatura peruana.$^{6}$ Porras ${ }^{7}$ subraya las deficiencias de las novelas de Dávalos, pero tiene el acierto de mencionar la influencia de los Episodios nacionales de Galdós. Antonio Cornejo Polar en subreve "Historia de la literatura del Perú republicano" alude concisamente a Dávalos y apunta que La Ciudad de los Reyes "tiene méritos nada desdeñables". Estas escasísimas menciones reafirman que no es ninguna exageración decir que conocemos mal nuestra propia literatura ${ }^{9}$.

La Ciudad de los Reyes marca un importante salto con respecto a las novelas más representativas del siglo XIX peruanó, como son El padre Horán o Aves sin nido. En efecto, obseryamos un manejp del lenguaje que dota al texto de un poder de sugerencia del que carecen los obras de Aréstegui o Clorinda Matto. Por otro lado, el diseño de sus personajes no obedece al maniqueísmo extremo de los autores antes mencionados. Se trata de personajes más ricos, con facetas contradictorias, con dudas y vacilaciones, y por tanto mucho mas verosímiles, especialmente según los códigos decimonónicos. Por estos medios consigue Dávalos evitar el escollo del melodramatismo que mediatiza los logros de

5 Lima: P.L. Villanueva, 1975, IV, 1180-1181.

6 Lima: Peisa, 1993.

7 Fuentes históricas peruanas, 513.

8 Historia de la literatura del Perú republicano.En Fernando Silva Santisteban (Ed.), Historia del Perú, Lima, Mejía Baca, 1980, VIII, 66.

9 Fue Francisco Carrillo en sus cursos sobre literatura peruana del siglo XIX, quien me hizo interesarme por esta novela de Dávalos. A Carrillo corresponde pues el mérito de ser el primero en percibir la importancia de la obra. Recientemente, escuché al escritor Oswaldo Reynoso comentarios favorables hacia otra novela de Dávalos, Manuel Pardo. Aunque pocos, la obra de Dávalos tiene pues algunos lectores perspicaces. 
El padre Horán o Aves sin nido, diseñando una historia ajena a esquematismos, susceptible de interesar al lector moderno no especializado.

La Ciudad de los Reyes puede ser catalogada como una novela realista. Sus modelos literarios son ante todo el Galdós novelizador de la historia en los célebres Episodios Nacionales, pero también las Novelas Contemporáneas del mismo autor (su reconstrucción de los ambientes limeños debe mucho al Galdós de, por ejemplo, Fortunata y Jacinta, y su minuciosa recreación del Madrid decimonónico), y más mediatizadamente, Balzac. Su estrategia narrativa se orienta a presentarnos un retrato de la sociedad limeña de fines del siglo pasado. La novela de Dávalos es indudablemente mucho mas fiel que la de Clorinda Matto a los postulados del realismo decimonónico. La escritora cuzqueña se plantea a nivel teórico producir un texto realista e incluso naturalista, pero el fuerte tinte melodramático lastra involuntariamente a Aves sin nido de rezagos románticos. Esto no sucede con la obra de Dávalos, en la cual los escasos elementos que se podrían tipificar de románticos no atentan contra el equilibrio del conjunto. El protagonista de la novela. Urzúa, a pesar de su amor imposible (debidamente refrenado) por Marta, no es de ningún modo un héroe romántico, como lo comprobamos en nuestro análisis. Pepe Cegama, el personaje más idealizado d la novela, se muestra sin embargo realista al aconsejar a su hermana Manuelita en cuantô à suș rellaciones con Florimbố. El episodio más romántico lo constituyen los amores contrariades de, Marta y Orbea, pero incluso al final Marta se arrepiente de su romántica decisión de encerrarse en un convento. Se logra así un relato rico en matices, alejado de cualquier tipo de efectismos. No debió ser ajeno a este afán de equilibrio y de depuración formal la influencia modernista, entonces en pleno auge en toda la literatura hispanoamericana, con su tendencia a huir de las efusiones sentimentales propias del romanticismo, y su preocupación por el cuidado del estilo.

El proyecto escritural de Dávalos y Lissón incorpora una serie de contenidos ideológicos, dotando a la novela de una intencionalidad que trasciende lo meramente estético. Al igual que Aréstegui y Clorinda Matto, se propone transmitir un mensaje, una enseñanza, una reflexión, pero sabe hacerlo de una manera mucho más sutil, evitando el panfletarismo en el que con frecuencia incurren los escritores cuzqueños. La ideología que sustenta a La Ciudad de los Reyes puede ser tipificada como racionalista. Ese racionalismo, de base 
positivista, informa un proyecto socio-político de regeneración nacional orientado a una modernización capitalista. Dicha propuesta parte de un diagnóstico de la realidad nacional, a partir de la recreación ficcional de un momento histórico de gran importancia, como es el de la coyuntura de 1895, engarzado con un retrato de la sociedad limeña de la época. Ese proyecto de racionalización se evidencia tanto a nivel de lo público (luchas políticas, clases sociales, reconstrucción económica), como de los privado (la familia, el matrimonío, la moral, la religión). En este estudio se abordará con mayor amplitud el nivel de lo público, y más escuetamente el nivel de lo privado.

\section{La esfera pública}

La acción de la obra, como lo hemos adelantado, se ubica en 1895, año marcado en la historia peruana por la revolución de Piérola. La novela recrea estos acontecimientos. Aunque Dávalos se toma algunas libertades con la historia, es fácil reconocer a los principales protagonistas de los hechos: el doctor Cayo es indudablemente Piérola, el Presiđente (al que nunca se asigna nombre) es Cáceres, y el doctor Abadía puede ser identificado con la figura de Candamo, el presidente del Partido Civil. En cuanto a los demás personajes, es inútil buscarles equivalentes reales, pues no se trata de una novela "en clave".

El ascenso al pöder de Piéroła, àpoyađôt por sus montoneras, marca una etapa en la historia peruanac Coneelliaseninicia el más largo periodo de democracia formal que ha conocido el país: la denominada República Aristocrática. Piérola, jefe del Partido Demócrata, sube al poder en alianza con el Civilismo, que usufructuará el mando en casi todo el resto del periodo. La importancia de la República Aristocrática, empero, no reside principalmente en su inusual estabilidad política. Se trata de un periodo en que se produce un importante desarrollo capitalista bajo hegemonía inglesa. Es la época de la reestructuración capitalista de las grandes haciendas azucareras del norte, del nuevo auge de la explotación minera y petrolera, del boom del caucho, de la incipiente industrialización de Lima. Es también el periodo de la reconstrucción nacional posterior a la guerra con Chile. Se refuerzan en el Perú las ideas liberales y positivistas, sustento ideológico necesario de todo proyecto de expansión capitalista. El racionalismo es uno de los ejes de este conjunto de ideas. En efecto, a ojos burgueses, el desarrollo capitalista implica cancelar una estructura social basada en una tradición que aparece como irracional (el sistema semi-feudal heredado de la Colonia). 


\subsection{Visión del momento histórico}

Dávalos y Lissón presenta en La Ciudad de los Reyes un Perú convulsionado por las pugnas intestinas, todavía incapaz de reponerse del desastre de la guerra del Pacífico. El régimen del Presidente, el héroe de la guerra, se ha hecho impopular debido a los abusos y latrocinios de sus allegados. El Civilismo, que lo había apoyado, le retira su respaldo y se alía con el doctor Cayo. El estallido de la lucha fratricida genera una situación de intranquilidad en el país, con el consiguiente perjuicio de la actividad económica en tiempos de reconstrucción nacional, y causa gran destrucción y mortandad, particularmente en Lima. Los combatientes caídos son principalmente campesinos, con frecuencia desconocedores de los móviles de la contienda "Había muerto por su patria; los caudillos del movimiento exigieron su sangre para la regeneración de ella; y él, sin averiguar si había lealtad en ese llamamiento... había dado lo único que poseía: su vida"10.

La labor del Presidente es emjuiciada con dureza, aunque reconociendo su honestidad personal y sus buenas intenciones: "dotado de un espíritu superior, no se manchó nunca haciendo ruindades... A pesar de haber sido malísimo gobernante, seguía creyéndose caudillo y predestinado para mandar la República. La verdad era, que a la sombra de su nombre, muchos de los suyos... habían exp̉otadoce tiranizaza al pueblo, y él sin saberlo pagaba esas ajenas culpas" (278-279). El sostén principal del régimen es el ejército, leal al presidente por su destacado papel en la guerra, pero el odio popular abre paso al ascenso del doctor Cayo y su Partido Demócrata (el episodio del sitio de Lima por los montoneras rebeldes es de los más logrado de la novela). Dávalos destaca el papel del doctor Cayo y en especial su audacia que, a la cabeza de sus montoneras, le valió la victoria, y asimismo el prestigio alcanzado por el caudillo en los sectores populares. El rol que le cabe es el de restablecer la legalidad y abrir paso (en las esperanzas de Dávalos) a una era de progreso para el país.

Sin embargo, a pesar de la crítica general a la casta política (a la que reprocha arbitrariedades y peculados), las simpatías del autor parecen inclinarse más bien hacia el Civilismo. Esto lo evidencian pequeñas alusiones,

10 Pedro Dávalos y Lissón, La Ciudad de los Reyes, Lima: Instituto Nacional de Cultura, 1989, 285. En adelante, todas las citas remiten a esa edición. Se indicará simplemente el número de página entre paréntesis. 
como las alabanzas dedicadas a Manuel Pardo, el fundador del Civilismo, por un típico militarista, el coronel Martínez: "Pardo fue un genio, y mientras no tengamos otro, no queda sino la gente de sable"(16); o las reflexiones de la señora Caridad Quinieros, cómplice del asesinato de Pardo, tardíamente conciente de las funestas consecuencias del crimen: "lo que más le preocupaba era la guerra con Chile, venida... porque Pardo había muerto; y perdida porque él y su partido no estuvieron al frente de la defensa" (215); también subrayan estas simpatías el papel del doctor Abadía, el político más capaz presentado en la novela, cuya segura visión anuncia a los representantes de Loreto la inutilidad de sus gestiones. Dávalos ve en el Civilismo, heredero de Pardo, el partido capaz de llevar adelante la reconstrucción nacional; a pesar de no ser un partido popular, es el que cuenta con gente más capacitada para una tarea de esa envergadura. Para él, sólo el Civilismo, contando con cuadros capaces y con un líder de la talla de Pardo, hubiera estado en condiciones de evitar el desastre de la guerra con Chile, pues no bastaba con el valor o la capacidad de algunos militares, sino que era necesario contar con un estado debidamente organizado.

Para Dávalos, la derrotaconstituye una vergüenza nacional, tal como lo piensa el protagonista de la novela, Urzúa: "Cinco años de guerra habían desmembrado, humillado y empobrecido a la patria; y cuando se esperaba que fueran el arrepentimiento, ta paz y el trabajoplos frutos de tan dura lección, aún no teníg dérming daicerrụpeción, las muerte, la ruina y el envilecimiento" (294).

Otro problema más se plasma en la conflictiva coyuntura política representada en la novela: las reinvindicaciones de Loreto, que en esos momentos, con el auge cauchero, aparecía como uno de los puntales del progreso del país. Dávalos reconoce el carácter asfixiante que ya entonces tenía el centralismo limeño y la justicia de las reinvidicaciones autonómicas loretanas, pero al mismo tiempo resalta la impopularidad de esa propuesta y el temor de una anexión de Loreto por el Brasil.

\subsection{El mundo representado. La sociedad limeña}

Dávalos pretende brindarnos en La Ciudad de los Reyes una imagen de la realidad peruana, y no es casual que este esfuerzo tengo como eje a Lima, como nos lo explica por boca del doctor Abadía: "El Perú es Lima, y 
en saliendo de Lima lo demás es anémico e incompleto en todo orden" (67). Para nuestro autor Lima expresa la conjunción de los factores positivos que deben permitir la regeneración del país: es el centro de la cultura, del progreso económico y de la legalidad. Del resto del Perú no nos dice mucho Dávalos. Nos da de la selva la imagen de un territorio inhóspito, pero abundante en riqueza cuya extracción sólo reclama un poco de audacia. En cuanto a la sierra, no parece darle mayor importancia; nos da la impresión de un desolado vacío entre la civilizada Lima y la promisoria selva.

Centremos pues nuestro análisis en la sociedad limeña. Dávalos presenta una visión crítica pero afectiva de Lima. La clase social a la que pertenecen los personajes de más relieve en la novela es la antigua aristocracia limeña. Urzúa proviene de una familia de este linaje, lo mismo que sus parientes, la familia Carcía Azuaga y la tía Peta; también son de esa extracción las familias Cegama, Orbea, Zúñiga. Pero esta aristocracia es ya una clase en decadencia. En efecto, perjudicadas por los avatares đe la turbulenta vida política o por el desastre de la guerra, la mayoria de familias de esa clase se encuentran económicamente arruinadas. Los que han logrado conservar su status lo han hecho en base al aprovechamiento inescrupuloso de los bienes del estado, o en contadas ocasiones, por su dedicación a tareas productivas que implican su conversión en una auténtica burguesía. Las familias Cegama y Otzuarte (a la que pertenece Peta) Prépresentą a las estirpesémpobrecidas, constituyendo un caso extremo la familia Zúniga, que incluso se ha visto obligada a domiciliarse en un barrio popular; pero también los Cegama, luego de la muerte de Pepe, el sostén de la familia, conocen momentos muy negros, viéndose obligados los hermanos menores a trabajar de obreros. La familia García, en cambio, mantiene su holgada posición al parecer debido a algunos malos manejos de don Juan de la Cruz, quien llegó a desempeñar cargos de ministro. Alonso Urzúa, huérfano de aristocrática familia, se ha convertido en un próspero burgués mediante su labor en la extracción del caucho.

Los otros sectores sociales de la ciudad sólo son presentados de manera colateral. Los estratos populares están ejemplificados en primer lugar por un conjunto de sirvientes, de raza negra, descedientes de esclavos y vinculados con las grandes familias por lazos tradicionales. Los negros que no se ocupan en estos menesteres se dedican por lo general a actividades como la delicuencia, la prostitución o la mendicidad. Otros grupos sociales se sitúan en el camino del progreso, como los inmigrantes europeos, consa- 
grados a la actividad comercial; es el caso del italiano Bartolo Cusini, vecino de los Zúñiga, que ha logrado una posición económica desahogada mediante las ganancias de su pulpería. En cuanto a la población indígena, cuya presencia por entonces en Lima era poco significativo, sólo figura como carne de cañón en los diversos bandos en conflicto.

En la novela no se hacen visibles los conflicto sociales. Las relaciones entre las clases dominantes y las dominadas en la sociedad limeña parecen ser plenamente armónicas. Sólo existen los problemas derivados de los abusos de algunos funcionarios, como el negociado con las pensiones de las viudas, o los causados por la violencia política. Los conflictos políticos no parecen tener ninguna relación con los intereses de clase, y toda rebelión social se vincula con la insania y la corrupción moral: es el caso de Samperio y de su padre, los únicos contestatarios de la novela, que son considerados como resentidos y como una lacra social, a pesar de las condiciones personales que pudieran tener.

\subsection{El proyecto nacional}

El proyecto nacional en el cual cree Dávalos y Lissón supone un decidido esfuerzo de modernización y progreso. Ya hemos señalado el sustento racionalista del proyecto. Dotar al país de una estructura racionalmente organizada y eficazmente fürigional constituye uri auténtico esfuerzo de regeneración nacional perentoriamente exigido por la debacle consecutiva a la guerra con Chile. Este esfuerzo implica el reordenamiento de el vida política, mediante el establecimiento de una legalidad democrático-liberal, basada en partidos sólidos, integrados por cuadros idóneos, capaces de garantizar la necesaria estabilidad administrativa. Esta estabilidad es el requisito indispensable para la transformación esencial que debe procesarse, la económica.

El problema principal de la vida nacional es la inoperancia de su sistema productivo. Aquí es donde ve Dávalos la necesidad de un cambio fundamental, cambio cuyo eje debe ser el trabajo. Ya no tiene cabida una clase parasitaria acostumbrada a vivir de la renta de la tierra, se impone la necesidad de un grupo social con espíritu de empresa, apto para lograr un reordenamiento capitalista de la economía. El país necesita crear más riqueza y por esto el proyecto de Dávalos implica una nueva valoración del trabajo, desde la perspectiva de quien invierte un capital y sabe sacar provecho de los recursos del país, tanto materiales como humanos. 
Así, al reflexionar Urzúa sobre el futuro de sus protegidos Lucas Cegama y Narciso Zúñiga, a quienes lleva consigo a Loreto, opina que: "de nada debían estar tan orgullosos, como de haber principiado codeándose con el pueblo y con los oficios del pueblo. Estaba convencido de que era necesario levantar a sus ojos el prestigio del trabajo, desacreditado en más de tres siglos de inicua esclavitud" (296). Pero el trabajo que se valora no es principalmente el del productor asalariado, sino el del empresario, que es visto como el factor dinamizador de la economía y el sujeto social impulsor del cambio. El engrandecimiento del país será fruto del espíritu de iniciativa de este sector, cuyas cualidades de laboriosidad y disciplina lo hacen apto para producir un reordenamiento de la estructura productiva y, en general, de la vida nacional: "en el Perú no habrá buena política, ni honradez administrativa, hasta que el poder no esté en manos de hombres independientes, de fortuna y de trabajo. Quien ha sabido administrar su hacienda propia, puede administrar la pública" (910), dice el próspero minero Moreno. Esta nueva clase, esta burguesía, debe conformarse fundamentalmente en base a los miembros de la antigua aristocracia, siempre que éstos sean capaces de superar su tradicional desprecio al trabajo y adoptar los nueyos valores. Si bien la visión de Lima de Dávalos no es pasatista, sí se identifica con algunos valores de las familias tradicionalmente dominantes, cuya supremacía piensa debe continuar, siempre y cuando se produzca en sus integrantes ese cambio de actitud.

\section{Biblioteca de Letras}

Las actividades que para Dávalos permitirán la transformación económica del país son ante todo las extractivas como el caucho o la minería, y también el comercio y la industria. En cambio, no parece tener mucha confianza en la producción agrícola, a la que ve como una actividad sin mayor futuro: Orbea por ejemplo se arruina con la agricultura y se ve forzado a dedicarse a la extracción del caucho, y Lecuona parece condenado a permanecer en la mediocridad de sus labores agrícolas. Esta actitud curiosamente se contrapone a la importancia decisiva que tuvo la agricultura en la recuperación económica que marcó a la República Aristocrática; no olvidemos que ésta fue la época de la expansión o reconstrucción de las grandes haciendas azucareras del norte.

Este proyecto responde, como lo apuntamos, a una visión del mundo, a una ideología de base positivista. Su meta es hacer del Perú un país moderno, es decir un país capitalista, capaz de sacar partido de sus abundantes recursos naturales y humanos. Por esto, adolece de limitaciones fácil- 
mente visibles hoy, propias del momento histórico y de los intereses sociales asumidos por Dávalos. En primer lugar, y esto es notorio y observable, Dávalos no tenía la menor conciencia de que la riqueza no se origina gracias a la capacidad administrativa de algunos empresarios, sino al trabajo de quienes realizan las tareas productivas: no tiene pues ninguna conciencia del fenómeno de la explotación. Es por esto que nos presenta la extracción del caucho como una actividad relativamente sencilla, ignorando los padecimientos y vejaciones que sufría el trabajador cauchero, y el despojo de los pueblos amazónicos (no es por mala suerte que Orbea muere de un flechazo).

Otros aspectos escapan también al esquema ideológico de Dávalos. El problema de la tierra y de la población indígena serrana no tiene cabida en la obra, y sin resolver ese problema era imposible transformar la economía nacional, dado que la estructura semi-feudal de la propiedad terrateniente serrana ha sido siempre un obstáculo a cualquier intento profundo de desarrollo capitalista. El otro elemento que no podía percibir es que el desarrollo capitalista que entonces empezaba a producirse se daba bajo dependencia de capitales foráneos, en ese momento principalmente ingleses.

Estas que ahora percibimos como limitaciones no deben hacernos olvidar que en ese periodo un proyecto de la naturaleza del que enarbolaba Dávalos, representabả unla propuesta decărácter progresivo, que de haber logrado ser realizada, hubiera hechemenos delorosa la historia del siglo XX peruano.

\section{La esfera privada}

La ideología racionalista que hemos detectado en el conjunto de la obra se manifiesta igualmente en la esfera de lo privado. El proyecto de racionalización de la vida nacional incluye también una reforma de la conducta personal de los peruanos. Esto lo podemos entrever al estudiar el comportamiento en este plano de los diversos personajes. Apuntaremos sintéticamente algunas observaciones sobre estos aspectos.

El matrimonio para Dávalos es más una cuestión de razón que de corazón, y a este respecto adquiere fundamental relevancia la opinión de la madre, que es la persona experimentada en la materia y que puede aconsejar a la hija. No se trata solamente de tener en cuenta los aspectos económicos de un enla- 
ce, importantes por sus consecuencias sobre la vida en común, sino también la necesaria compatibilidad de caracteres. Se trata de dotar a la joven con un marido capaz de garantizarle seguridad y respeto, antes que amor pasional. Para el marido, se trata de conseguir una digna madre para sus hijos, y en lo posible, una buena aportación a la economía familiar. El amor mutuo no es lo decisivo en una relación de esta índole, y menos aún la pasión desmedida, tan valorada por los románticos. En La Ciudad de los Reyes se plantean reiteradamente los problemas que conlleva la elección de la pareja, y en particular el conflicto entre la voluntad de la madre y la de la hija. Dávalos valora positivamente la obediencia a la figura materna, cuya experiencia y madurez debe prevalecer sobre los caprichos pasajeros de los hijos.

A este nivel, adquiere una dimensión descollante la figura de la señora Rosa, presentada como la madre de familia sabia y prudente, incluso valerosa cuando se trata de defender su hogar, en contraposición a su marido, don Juan de la Cruz García, hombre de pocas luces, siempre al borde de cometer un error político, a no ser por el oportuno consejo de su esposa. Doña Rosa es la verdadera matriarca de la familia García Azuaga, y su perspicaz opinión debe ser considerada al tratar cualquier enlace. Siguiendo su consejo se casa felizmente su hija Lía, y su palabra es determinante para convencer a Beatriz de casarse con el señor Leniz, luego de la muerte de su amado Pepe Cegama; logra también arrancar a su hija Raquel de unamorireprobable per Samperio. Por el contrario, cuando su opinión es desoída, se producen consecuencias funestas. Su hijo Abel, casado por despecho contra la voluntad de su madre, se automargina del círculo social al que pertenece. Marta, encerrada también por despecho en un convento por no acatar la voluntad materna, se arrepiente finalmente de su desgraciada reclusión. Se hace aquí palpable el antirromanticismo de Dávalos: las pasiones tumultuosas son valoradas negativamente por atentar contra la unidad familiar. Esto es visible también en el caso de Manuelita, que finalmente se casa con Florimbó a pesar del engaño de éste (lo que en el romanticismo hubiera producido una sangrienta escena de celos); y el prudente Alfonso Urzúa sabe refrenar su imposible amor por Marta (imaginemos el espectacular rapto de la monja de clausura en una novela romántica).

Finalmente, examinemos el enfoque de la religión. Para Dávalos y Lissón, la verdadera religión es entendida como ajena a toda suerte de fanatismo, superstición o misticismo. Se trata de una fe razonable y razonada. Por esto Dávalos es hostil a la beatería, contra la que enfila sus críticas. Así, 
ridiculiza a Peta en el caso de la supuesta santa Marcela, a cuyo pañuelo se atribuyen virtudes milagrosas, resultando la presunta santa una impostora. A la credulidad de Peta se opone la cordura de su siempre ponderada prima Rosa, desconfiante de esos prodigios, o de su sobrino Alfonso, quien llega a jugar una atrevida broma a su tía Peta, y también de Monseñor Zavaleta, paradigma de una religiosidad centrada y cuerda, y crítico de las beatas que pretenden entronizar santos al margen de la jerarquía eclesiástica. La religión no debe pues ser fuente de excesos, sino apoyo de la moral y consuclo de las adversidades humanas.

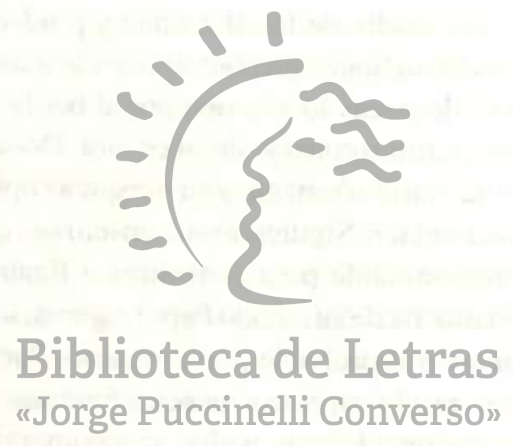

